\begin{tabular}{ll} 
POS & PROCEEDINGS \\
\hline
\end{tabular}

\title{
Catching Up on State Transitions in Cygnus X-1
}

\author{
Moritz Böck, Manfred Hanke, Jörn Wilms, Stefan Pirner \\ Dr. Karl Remeis-Observatory, Bamberg \\ Erlangen Centre for Astroparticle Physics, Germany \\ E-mail: Moritz.Boeck@sternwarte.uni-erlangen.de
}

\section{Victoria Grinberg, Sera Markoff}

Astronomical Insitute “Anton Pannekoek”, University of Amsterdam, Netherlands

\section{Katja Pottschmidt}

CRESST and NASA Goddard Space Flight Center

Center for Space Science and Technology, University of Maryland Baltimore County

\section{Michael A. Nowak}

Massachusetts Institute of Technology, Kavli Institute for Astrophysics and Space Research, CXC

\section{Guy Pooley}

Cavendish Laboratory, Cambridge, UK

In 2005 February we observed Cygnus X-1 over a period of 10 days quasi-continuously with the Rossi X-ray Timing Explorer and the Ryle telescope. We present the results of the spectral and timing analysis on a timescale of $90 \mathrm{~min}$ and show that the behavior of Cyg X-1 is similar to that found during our years long monitoring campaign. As a highlight we present evidence for a full transition from the hard to the soft state that happened during less than three hours. The observation provided a more complete picture of a state transition than before, especially concerning the evolution of the time lags, due to unique transition coverage and analysis with high time resolution.

VII Microquasar Workshop: Microquasars and Beyond September 1-5 2008

Foça, Izmir, Turkey 


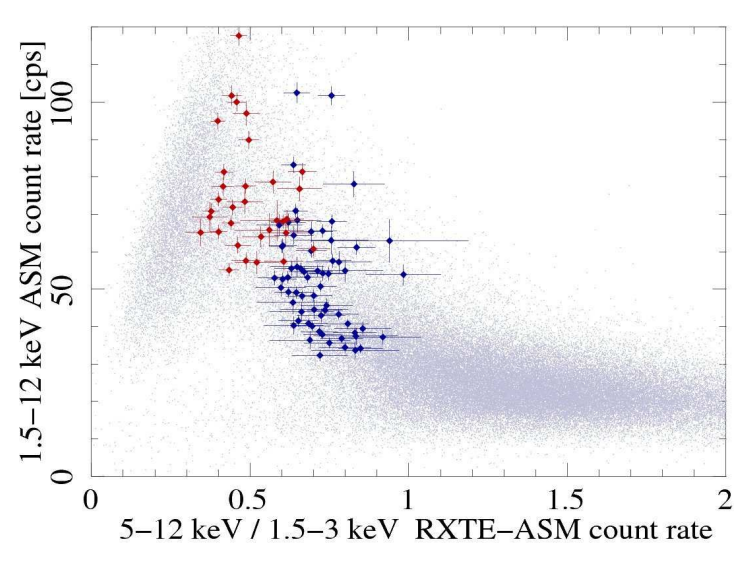

Figure 1: Hardness-intensity-diagram of Cyg X-1 from $R X T E$-ASM. The gray dots show the long term behaviour of Cyg X-1 from 1996 January until 2008 July by single 90 min dwells. The hard (right) and soft (left) states are clearly separated. The density of dots measures how much time the source spends in each state. The red (soft-intermediate) and blue (hard-intermediate) data points were obtained within a 10 day period and show the transition in 2005 February, which is the topic of this paper.

\section{Introduction}

The microquasar Cygnus X-1 is usually observed in the low/hard state or in the high/soft (steep power-law) state - or in an intermediate state [10].

In the low/hard state the 4-250 keV RXTE broadband spectrum can be described by a broken power-law (with $\Gamma \equiv \Gamma_{1} \lesssim 2.1^{1}$ for $E<E_{\text {break }} \approx 10 \mathrm{keV}$ and $\Gamma \equiv \Gamma_{2}<\Gamma_{1}$ for $E>E_{\text {break }}$ ) with high-energy cutoff $\propto \exp \left(-E / E_{\text {fold }}\right)$ (with $120 \mathrm{keV} \lesssim E_{\text {fold }} \lesssim 250 \mathrm{keV}$ ) [10], which is canonically interpreted as the result of Comptonization by thermal electrons. The variability is large $(\approx 30 \%$ root mean square (rms) amplitude) due to several broad noise components in the power spectrum (PSD) between 0.01 and $100 \mathrm{~Hz}$, which can be modeled with Lorentzian profiles [7, 9]. In the hard state Cyg X-1 emits a flat radio spectrum with a flux of $\sim 15 \mathrm{mJy}$ [4].

In the soft state the soft X-ray spectrum is dominated by thermal emission from the accretion disk, which is believed to extend close to the innermost stable orbit around the black hole. The power-law component of the spectrum is steeper, but often extends into the $\gamma$-ray regime without notable cutoff, indicating that Comptonization by non-thermal electrons is much more important in the soft state than in the hard state [6,3, see also the contribution of J. Malzac to this volume]. The radio emission in the soft state is quenched. The timing properties are also very different: the PSD is a $f^{-1}$ powerlaw with cutoff at $\approx 10 \mathrm{~Hz}$, without the broad noise components and thus a much lower variability [9].

In the intermediate state X-ray and radio flares can be observed [11]. Cyg X-1 often shows failed state transitions instead of full transitions [8]. To determine the nature of the transitions it is helpful to combine spectral and timing properties. The peak frequencies of the Lorentzians in the power spectra are correlated with the photon index $[9,1]$. Time lags between light curves in different energy bands depend on the photon index as well, the correlations can be explained with models based on Comptonization in a jet [5]. How some of these correlations change after the transition is discussed in this work.

\section{Observation and Data Analysis}

In 2005, we observed Cyg X-1 quasi-continously with the Rossi X-ray Timing Explorer ( $R X T E$ ) from Feb 1, 10:48 to Feb 4, 7:11 UTC (MJD 53402.45-53405.3) and from Feb 7, 0:21 to Feb 10,

1 A definition of the typical hard state spectrum is given by $\Gamma_{1} \leq 2.1$ [10]. Note that the transition from the intermediate hard to the intermediate soft state, which is analyzed in this work, occurs at $\Gamma_{1} \approx 2.4$. 

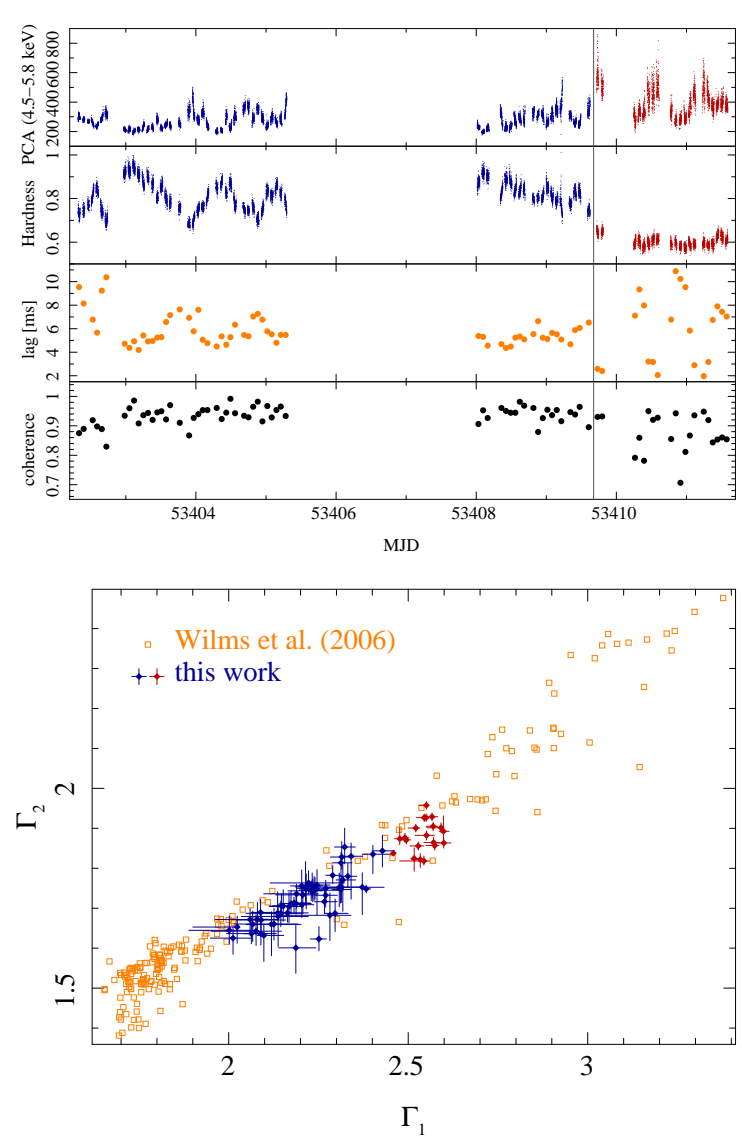

Figure 2: Temporal behavior of the count rate, the hardness, the time lag, and the coherence. The latter two were computed between the $9.5-15 \mathrm{keV}$ and 4.5-5.8 keV light curves. The vertical line indicates the transition from the hard-intermediate to the softintermediate state. A positive time lag means that the high energy light curve has a delay with respect to the one in the low energy band.

Figure 3: Relation between the photon indices. The linear correlation found in this work is identical to that found in the long term spectral evolution from 1999-2004 [10]. This correlation holds throughout all states. The transition occured at $\Gamma_{1}=2.4$.

14:14 UTC (MJD 53408.01-53411.59), and the Ryle telescope. The detailed results of our analysis will be described by Böck et al. (2009) [2]. A hardness-intensity diagram from RXTE-ASM data (Fig. 1) already shows that Cyg X-1 was in an intermediate state during this time. Because of the fast and strong variability due to flaring, we split the data into single $R X T E$ orbits of $\sim 3 \mathrm{ks}$ exposure.

For each of these orbits, we extracted the spectrum and PCA light curves in the 4.5-5.8 keV and 9.5-15 keV energy bands. Using these light curves PSDs were computed, which we call PSD $_{\text {lo }}$ and $\mathrm{PSD}_{\mathrm{hi}}$ in the following. Between these light curves we calculated coherence and time lags [7]. Consistent with previous observations the $9.5-15 \mathrm{keV}$ light curve lags the one at lower energies $[7,9]$.

The spectra were fitted with absorbed broken power-law models with exponential cutoff. During this observation, the photon index varied in the range $2 \leq \Gamma_{1} \leq 2.6$, which is more than a third of the range $1.65 \leq \Gamma_{1} \leq 3.4$ found for the long term evolution of Cyg X-1 from 1999 to 2004 [10]. The same relation between the two photon indices was found (Fig. 3).

We fitted the power spectra with two broad Lorentzian profiles $\left(\mathrm{L}_{1}\right.$ and $\left.\mathrm{L}_{2}\right)$ and an additional power-law with exponential cutoff where required, similarly to [1].

\section{Correlation of the spectral and timing properties}

In comparison with a PSD from a spectrally harder state (Fig. 4, left), $\mathrm{L}_{1}$ and $\mathrm{L}_{2}$ are shifted to higher frequencies in a PSD corresponding to a spectrally softer state (Fig. 4, right). Furthermore 

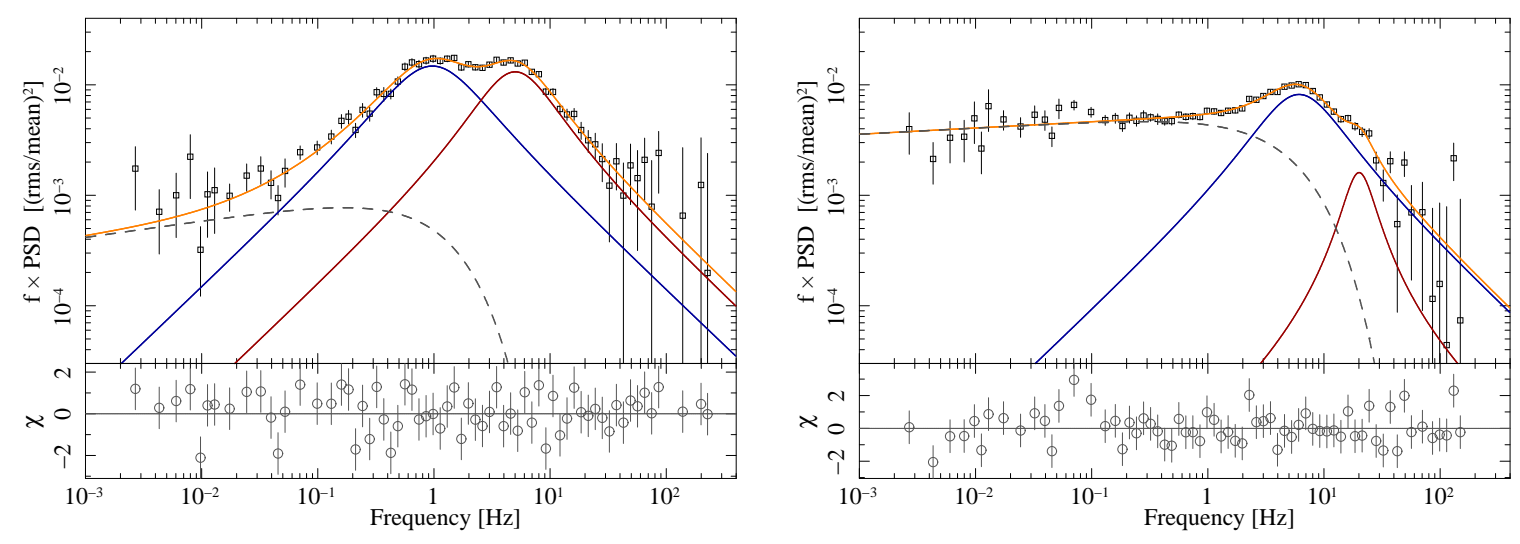

Figure 4: $\mathrm{A} \mathrm{PSD}_{\mathrm{lo}}$ at $\Gamma_{1}=2.14$ (left) and a $\mathrm{PSD}_{\mathrm{lo}}$ at $\Gamma_{1}=2.55$ (right), modeled as sum of two Lorentzian profiles ( $\mathrm{L}_{1}$ : blue curve, $\mathrm{L}_{2}$ : red curve), and a power-law with exponential cutoff (gray dashed line).
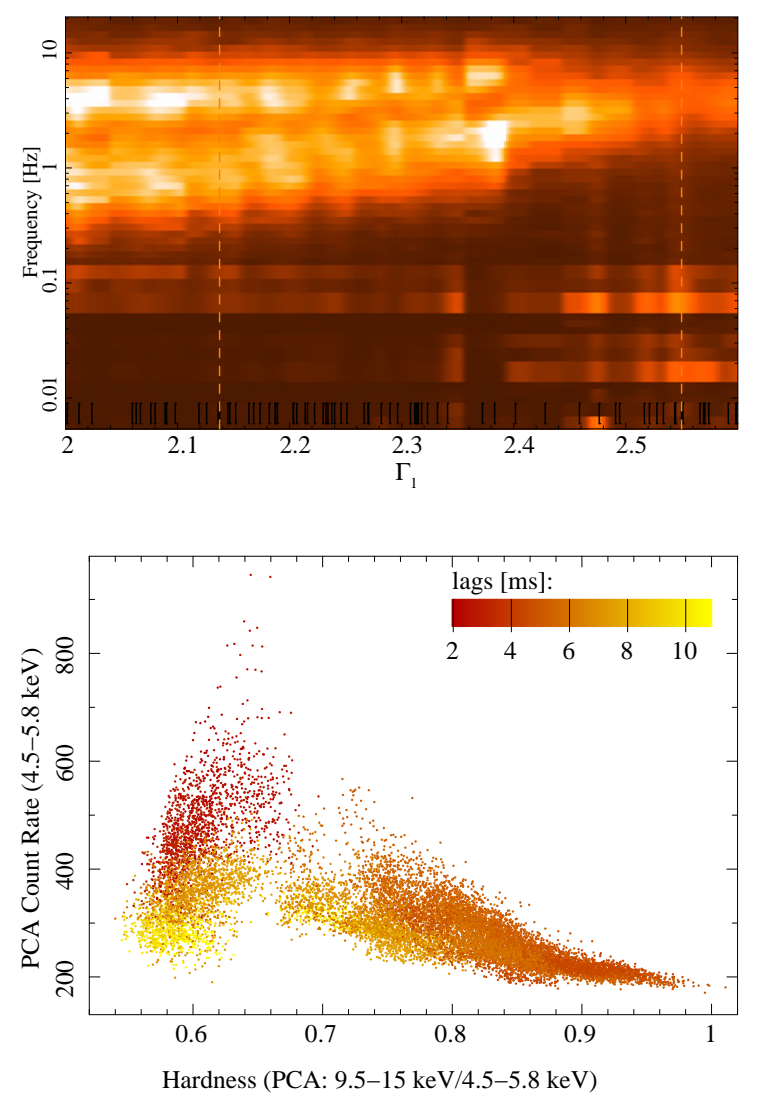

Figure 5: $\mathrm{PSD}_{\mathrm{lo}}$ as a function of $\Gamma_{1}$ : For each observation (black dashes at the bottom), the values of $f \times \operatorname{PSD}_{\mathrm{lo}}(f)$ are color-coded (brighter for larger values) and interpolated between the corresponding photon indices. The vertical dashed lines mark the PSDs shown in Fig. 4. The two bright streaks between $\sim 0.5$ and $\sim 10 \mathrm{~Hz}$ are the peaks of $\mathrm{L}_{1}$ and $\mathrm{L}_{2}$ in the PSDs, which obviously shift to higher frequencies with increasing $\Gamma_{1}$. After the transition at $\Gamma_{1} \approx 2.4, \mathrm{~L}_{2}$ fades and a $\mathrm{PSD} \propto f^{-1}$ power-law component emerges at low frequencies.

Figure 6: The hardness intensity diagram reveals a state transition. After the transition (see Fig. 2), the values cover a region in the HID separated from that with larger hardness before. The HID is colorcoded with the values of the time lags. In the soft region we found an interesting relation between the time lags and the count rate. A small time lag at high count rates and a large one at low count rates is observed. It suggests to subdivide this region into different states.

the normalization of the Lorentzians, especially that of $\mathrm{L}_{2},-$ and therefore the total variability of the source - decreases significantly, while the contribution of the power-law increases. This effect is obvious from the color-coded representation of the PSDs as a function of the photon index $\Gamma_{1}$ (Fig. 5). A quantitative analysis of the peak frequencies $v_{i}$ and fractional rms contribution $\mathrm{rms}_{i}$ of the Lorentzians $i=1,2$ proves these correlations. It is interesting that $v_{2}$ is roughly proportional to $\sqrt{v}_{1}$, especially when only the data from the hard-intermediate state are used.

A comparison of PSDs in the different energy bands reveales that the normalization of $L_{1}$ is 
significantly smaller in $\mathrm{PSD}_{\mathrm{hi}}$ than in $\mathrm{PSD}_{\mathrm{lo}}$, whereas $L_{2}$ is identical in both energy bands.

An interesting behavior of the time lags was found. In the hard-intermediate state they increase with decreasing hardness of the spectrum, as it was found in [9] and modeled in [5]. After the transition to the soft state there is a different behavior. The time lags change with the count rate. At high count rates there is a very low time lag $(\sim 2 \mathrm{~ms})$ and the light curves in the low and high energy band are strongly coherent, whereas it is the other way around at low count rates (Fig. 2 and Fig. 6).

\section{Acknowledgments}

We thank the organizing committees for this wonderful conference in Foça/Izmir, Turkey! This work was partially funded by the DLR (Deutsches Zentrum für Luft- und Raumfahrt) under contract 50OR0701.

\section{References}

[1] M. Axelsson, L. Borgonovo, and S. Larsson, Evolution of the 0.01-25 Hz power spectral components in Cygnus X-1, A\&A 438, 999-1012 (2005).

[2] M. Böck et al., Spectro-timing analysis of Cygnus X-1 during a state transition, A\&A (2009), in preparation.

[3] M. Cadolle Bel, P. Sizun, A. Goldwurm, J. Rodriguez, P. Laurent, A. A. Zdziarski, L. Foschini, P. Goldoni, C. Gouiffès, J. Malzac, E. Jourdain, and J.-P. Roques, The broad-band spectrum of Cygnus X-1 measured by INTEGRAL, A\&A 446, 591-602 (2006).

[4] R. P. Fender, G. G. Pooley, P. Durouchoux, R. P. J. Tilanus, and C. Brocksopp, The very flat radio-millimetre spectrum of Cygnus X-1, MNRAS 312, 853-858 (2000).

[5] N. D. Kylafis, I. E. Papadakis, P. Reig, D. Giannios, and G. G. Pooley, A jet model for Galactic black-hole X-ray sources: some constraining correlations, A\&A 489, 481-487 (2008).

[6] M. L. McConnell, A. A. Zdziarski, K. Bennett, H. Bloemen, W. Collmar, W. Hermsen, L. Kuiper, W. Paciesas, B. F. Phlips, J. Poutanen, J. M. Ryan, V. Schönfelder, H. Steinle, and A. W. Strong, The Soft Gamma-Ray Spectral Variability of Cygnus X-1, ApJ 572, 984-995 (2002).

[7] M. A. Nowak, B. A. Vaughan, J. Wilms, J. B. Dove, and M. C. Begelman, Rossi X-Ray Timing Explorer Observation of Cygnus X-1. II. Timing Analysis, ApJ 510, 874-891 (1999).

[8] K. Pottschmidt, J. Wilms, M. A. Nowak, W. A. Heindl, D. M. Smith, and R. Staubert, Temporal evolution of X-ray lags in Cygnus X-1, A\&A 357, L17-L20 (2000).

[9] K. Pottschmidt, J. Wilms, M. A. Nowak, G. G. Pooley, T. Gleissner, W. A. Heindl, D. M. Smith, R. Remillard, and R. Staubert, Long term variability of Cygnus X-1. I. X-ray spectral-temporal correlations in the hard state, A\&A 407, 1039-1058 (2003).

[10] J. Wilms, M. A. Nowak, K. Pottschmidt, G. G. Pooley, and S. Fritz, Long term variability of Cygnus X-1. IV. Spectral evolution 1999-2004, A\&A 447, 245-261 (2006).

[11] J. Wilms, K. Pottschmidt, G. G. Pooley, S. Markoff, M. A. Nowak, I. Kreykenbohm, and R. E. Rothschild, Correlated Radio-X-Ray Variability of Galactic Black Holes: A Radio-X-Ray Flare in Cygnus X-1, ApJ 663, L97-L100 (2007). 